\title{
Aspect Ratio Dependence of Heat Transfer in a Cylindrical Rayleigh-Bénard Cell
}

\author{
Guenter Ahlers, ${ }^{4,1}$ Eberhard Bodenschatz $\odot,{ }^{1,3,7,8}$ Robert Hartmann $\odot{ }^{2}$ Xiaozhou He $\odot,{ }^{6,1}$ Detlef Lohse $\odot,{ }^{2,3,1}$ \\ Philipp Reiter $\odot,{ }^{1}$ Richard J. A. M. Stevens $\odot,{ }^{2}$ Roberto Verzicco, ${ }^{5,9,2}$ Marcel Wedi, ${ }^{1}$ Stephan Weiss $\odot,{ }^{1,3}$ \\ Xuan Zhang $\odot,{ }^{1}$ Lukas Zwirner®, ${ }^{1}$ and Olga Shishkina ${ }^{1, *}$ \\ ${ }^{1}$ Max Planck Institute for Dynamics and Self-Organization, 37077 Göttingen, Germany \\ ${ }^{2}$ Physics of Fluids Group, J. M. Burgers Center for Fluid Dynamics and MESA+ Institute, \\ University of Twente, 7500 AE Enschede, Netherlands \\ ${ }^{3}$ Max Planck-University of Twente Center for Complex Fluid Dynamics, 7500 AE Enschede, Netherlands \\ ${ }^{4}$ Department of Physics, University of California, Santa Barbara, California 93106, USA \\ ${ }^{5}$ Dipartimento di Ingegneria Industriale, University of Rome "Tor Vergata," Via del Politecnico 1, Roma 00133, Italy \\ ${ }^{6}$ School of Mechanical Engineering and Automation, Harbin Institute of Technology, Shenzhen, 518055 China \\ ${ }^{7}$ Institute for the Dynamics of Complex Systems, Georg-August-University Göttingen, 37073 Göttingen, Germany \\ ${ }^{8}$ Laboratory of Atomic and Solid-State Physics and Sibley School of Mechanical and Aerospace Engineering, \\ Cornell University, Ithaca, New York 14853, USA \\ ${ }^{9}$ Gran Sasso Science Institute-Viale F. Crispi, 767100 L'Aquila, Italy
}

(Received 20 April 2021; accepted 13 January 2022; published 24 February 2022)

\begin{abstract}
While the heat transfer and the flow dynamics in a cylindrical Rayleigh-Bénard (RB) cell are rather independent of the aspect ratio $\Gamma$ (diameter/height) for large $\Gamma$, a small- $\Gamma$ cell considerably stabilizes the flow and thus affects the heat transfer. Here, we first theoretically and numerically show that the critical Rayleigh number for the onset of convection at given $\Gamma$ follows $\operatorname{Ra}_{c, \Gamma} \sim \operatorname{Ra}_{c, \infty}\left(1+C \Gamma^{-2}\right)^{2}$, with $C \lesssim 1.49$ for Oberbeck-Boussinesq $(\mathrm{OB})$ conditions. We then show that, in a broad aspect ratio range $(1 / 32) \leq \Gamma \leq 32$, the rescaling $\mathrm{Ra} \rightarrow \mathrm{Ra}_{\ell} \equiv \mathrm{Ra}\left[\Gamma^{2} /\left(C+\Gamma^{2}\right)\right]^{3 / 2}$ collapses various OB numerical and almost-OB experimental heat transport data $\mathrm{Nu}(\mathrm{Ra}, \Gamma)$. Our findings predict the $\Gamma$ dependence of the onset of the ultimate regime $\operatorname{Ra}_{u, \Gamma} \sim\left[\Gamma^{2} /\left(C+\Gamma^{2}\right)\right]^{-3 / 2}$ in the OB case. This prediction is consistent with almost-OB experimental results (which only exist for $\Gamma=1,1 / 2$, and 1/3) for the transition in OB RB convection and explains why, in small- $\Gamma$ cells, much larger Ra (namely, by a factor $\Gamma^{-3}$ ) must be achieved to observe the ultimate regime.
\end{abstract}

DOI: 10.1103/PhysRevLett.128.084501

Physics is abstraction, often assuming systems of infinite size. In the real world, this is not possible and finite-size effects come into play and thus must be understood. Here we will do so for the Rayleigh-Bénard convection (RBC), which has always been the most paradigmatic system to study buoyancy driven heat transfer in turbulent flow [1-3], which is of great importance in geophysical flows and in industry. The dimensionless control parameters are the Rayleigh number, the Prandtl number, and the aspect ratio $\Gamma$ of the cell, defined, respectively, as

$$
\mathrm{Ra} \equiv \alpha g \Delta H^{3} /(\kappa \nu), \quad \operatorname{Pr} \equiv \nu / \kappa, \quad \Gamma \equiv D / H,
$$

where $H$ and $D$ are the height and diameter of the cylindrical cell, $\alpha$ is the isobaric thermal expansion

Published by the American Physical Society under the terms of the Creative Commons Attribution 4.0 International license. Further distribution of this work must maintain attribution to the author(s) and the published article's title, journal citation, and DOI. Open access publication funded by the Max Planck Society. coefficient, $\nu$ is the kinematic viscosity, $\kappa$ is the thermal diffusivity, $g$ is the gravitational acceleration, and $\Delta \equiv T_{b}-$ $T_{t}$ is the temperature difference between the hot bottom plate and the cold top plate. The boundary conditions (BCs) are no-slip at all walls and the sidewalls are adiabatic. Within the Oberbeck-Boussinesq (OB) approximation, the flow dynamics for the velocity $\mathbf{u}$, the temperature $T$, and the kinematic pressure $p$ is given by the continuity equation $\nabla \cdot \mathbf{u}=0$ and the Navier-Stokes and convection-diffusion equations

$$
\begin{gathered}
\partial_{t} \mathbf{u}+\mathbf{u} \cdot \nabla \mathbf{u}+\nabla p=\nu \nabla^{2} \mathbf{u}+\alpha g T \mathbf{e}_{z}, \\
\partial_{t} T+\mathbf{u} \cdot \nabla T=\kappa \nabla^{2} T .
\end{gathered}
$$

The key response parameter is the Nusselt number (the dimensionless heat transfer)

$$
\mathrm{Nu} \equiv \frac{\left\langle u_{z} T\right\rangle_{z}-\kappa \partial_{z}\langle T\rangle_{z}}{\kappa \Delta / H}=\frac{H}{\kappa \Delta}\left\langle u_{z} T\right\rangle+1
$$


(a)

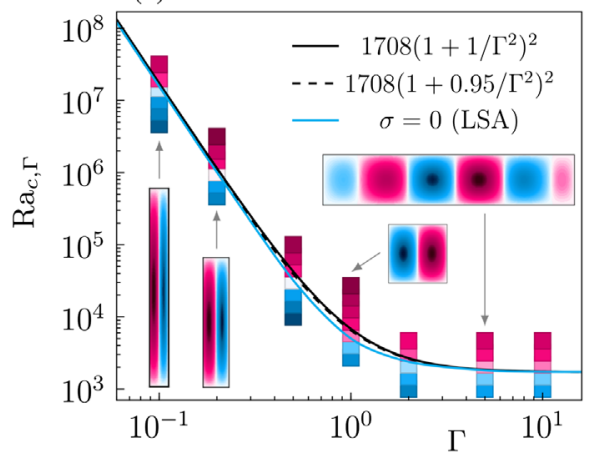

(b)

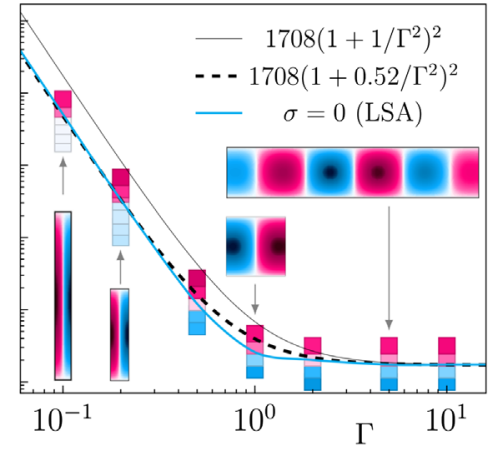

(c)

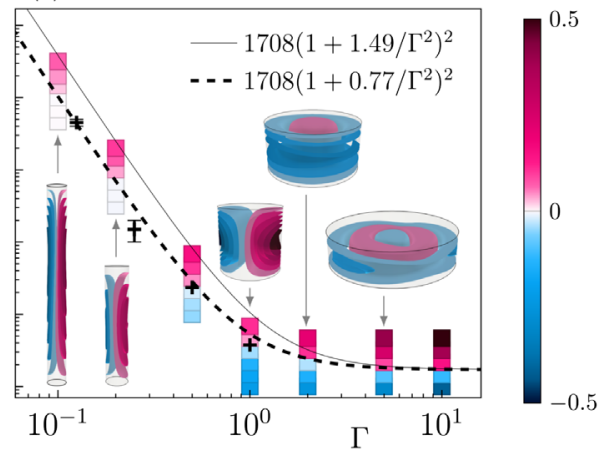

FIG. 1. Critical $\mathrm{Ra}_{c, \Gamma}$ for the onset of convection: Linear growth rates (colored vertically elongated boxes) from the linearized DNS approach (GOLDFISH) compared to the neutral stability curves (blue lines) from the eigenvalue LSA for (a) 2D box with isothermal sidewalls, (b) 2D box with adiabatic sidewalls, and (c) cylinder with adiabatic sidewall. Black lines show $\operatorname{Ra}_{c, \Gamma}=1708\left(1+C / \Gamma^{2}\right)^{2}$ with a best-fit $C$ for the linearized DNS data (dashed lines) and with theoretical $C$ for isothermal sidewall (solid line). Pluses in (c) show $\mathrm{Ra}_{c, \Gamma}$ from the nonlinearized DNS data (AFiD) [4]. Temperature contours near the onset of convection are shown for some $\Gamma$, as obtained from the linearized DNS. See details in [5-8] and the Supplemental Material [9].

where $\langle\cdot\rangle_{z}$ denotes the average in time and over a horizontal cross section at height $z$ from the bottom and $\langle\cdot\rangle$ is the time and volume average.

One key question-clearly, since Kraichnan's 1962 prediction of an ultimate regime [13-15] (i.e., the asymptotic law of heat transport at fixed $\mathrm{Pr}$ and extremely large $\mathrm{Ra}$ ) - is, what is the $\mathrm{Nu}(\mathrm{Ra})$ dependence for very large Ra? However, achieving very large Ra and thus this predicted ultimate regime is challenging, both experimentally, as large-scale setups are required, and computationally, as the number of grid points that can be handled is limited, too. Driven by the aim to nonetheless achieve very large $\mathrm{Ra}$, one is tempted to perform experiments or simulations at as small $\Gamma$ as possible. For a profound judgement on this, a
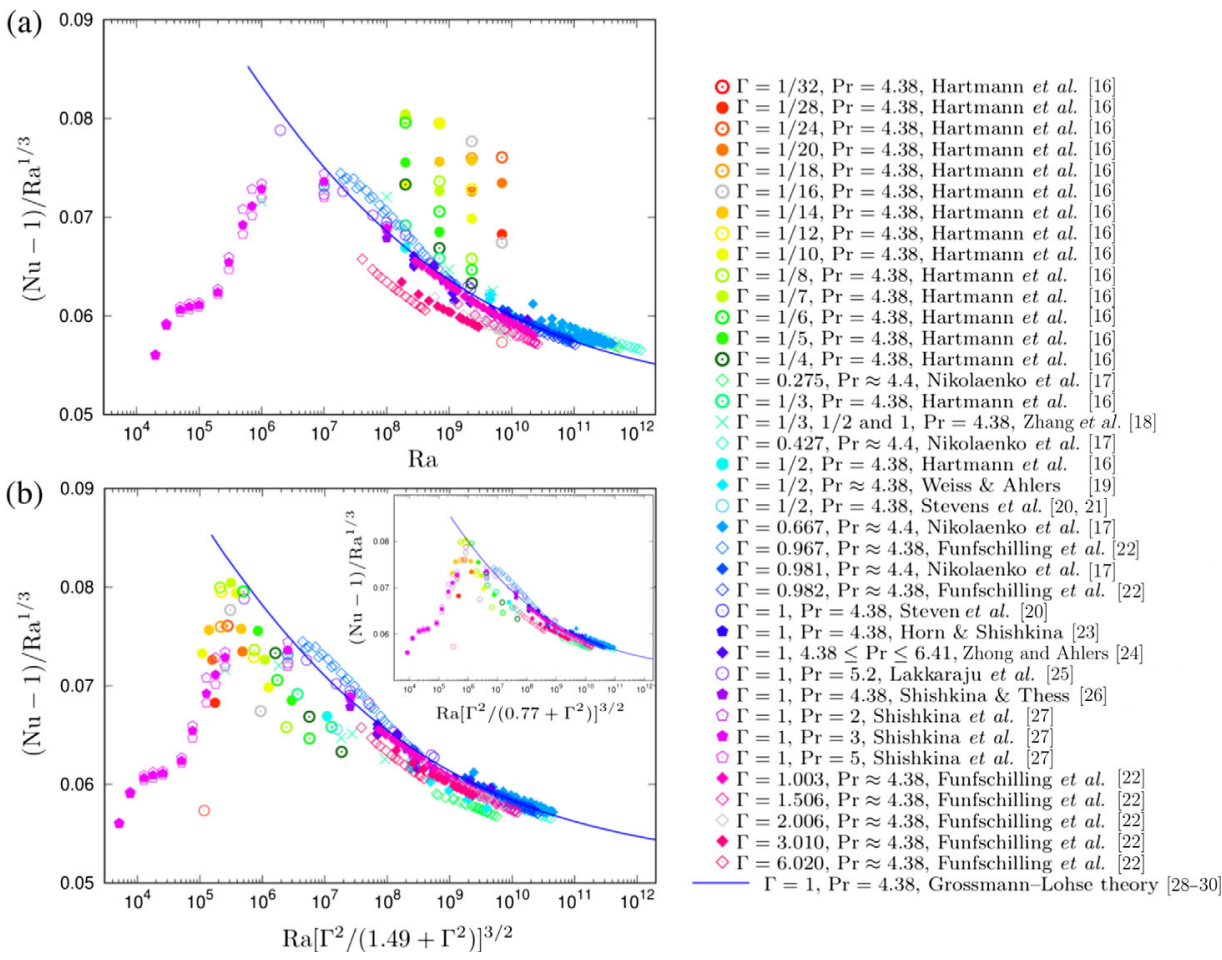

FIG. 2. (a) Compensated $\mathrm{Nu}$ vs Ra, as obtained in OB experiments and DNSs of RBC in a cylinder for Pr $\approx 4.4$ (water) and different $\Gamma$. Most data are for $\Gamma=1$ and $1 / 2$, which form the shape of this dependence. The data for extremely small $\Gamma$ show no discernible dependence. (b) Compensated $\mathrm{Nu}$ vs Ra based on the proper length scale $\ell$, for the same data as in (a). In the main plot, the theoretical value of $C=1.49$ is taken, while in the inset $C=0.77$, which corresponds to the best fit of the critical $\mathrm{Ra}_{c, \infty}$ for the onset of convection. Now the data for extremely small $\Gamma$ follow the general trend. 

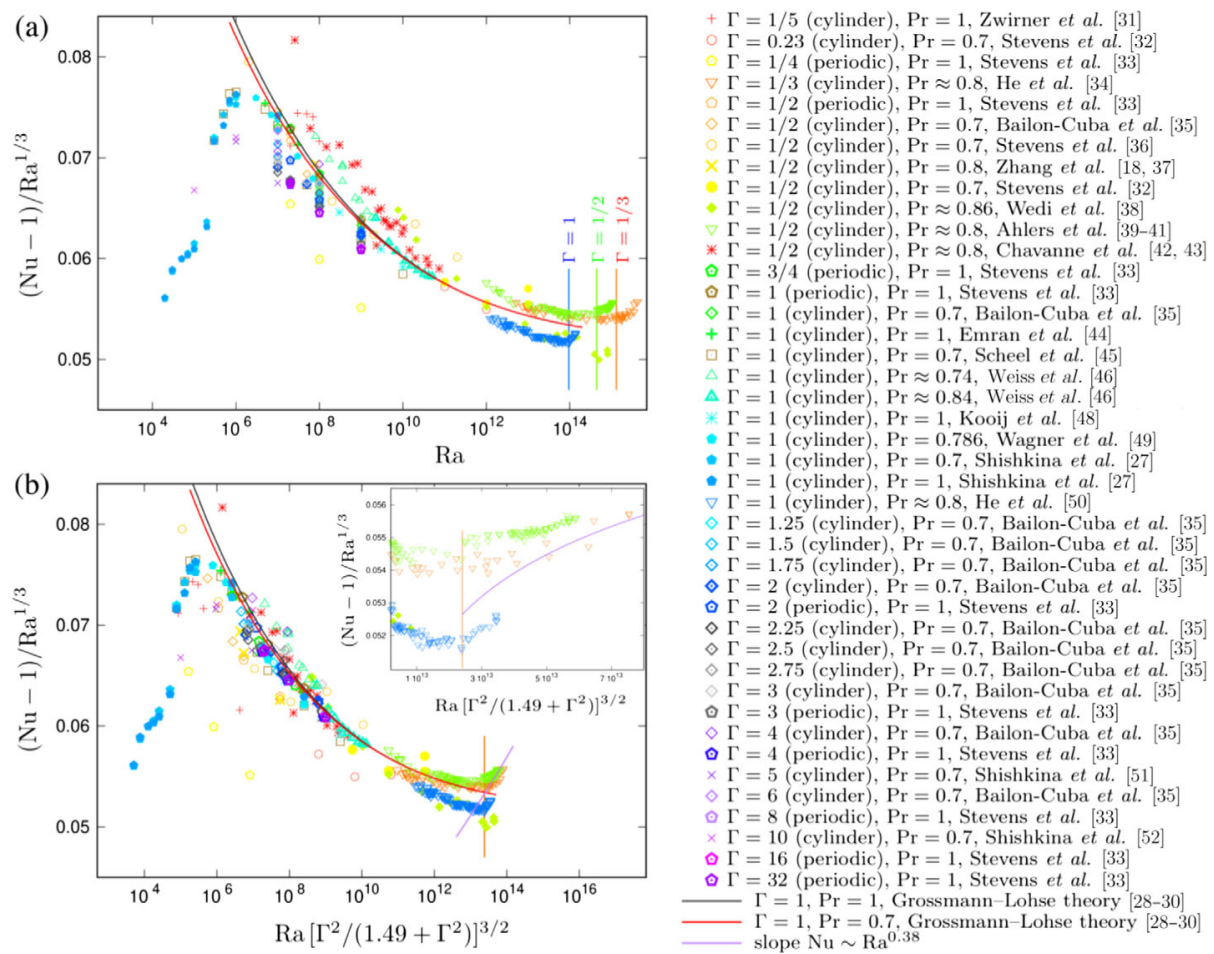

FIG. 3. (a) Compensated Nu vs Ra for OB RBC in a cylinder for Pr near 0.8 and in a 3D cell with periodic $\mathrm{BCs}$ for Pr $=1$ and different $\Gamma$. Vertical lines indicate the onset of the transition at high Ra, observed in Göttingen experiments (the onset moves to higher Ra with decreasing $\Gamma$ ). (b) Compensated $\mathrm{Nu}$ vs Ra based on the proper length scale $\ell$, for the same data as in (a). Now the transition happens at the same location for all $\Gamma$ [the vertical lines from (a) merge into one line]. The here presented experimental data from Chavanne $e t$ al. $[42,43]$ hold $\delta \rho / \rho<0.2$ for the density variation and $\delta \kappa / \kappa<0.2$ for the thermal diffusivity variation, as well as $\alpha \Delta<0.2$ and $0.68 \leq \operatorname{Pr} \leq 1$, i.e., similar almost-OB conditions as in [34,39-41,50] (however, in [34,39-41,50] the upper bounds for the fluid parameter variations are even slightly stricter). Data for $\mathrm{Pr}=0.74\left(\right.$ gas $\mathrm{N}_{2}$ ) and $\mathrm{Pr}=0.84$ (gas $\mathrm{SF}_{6}$ ) were taken using the same apparatus as in [47] but were not published there. The inset shows an enlargement at the highest Ra in normal representation for both axes (see also Supplemental Material [9], which includes [10-12]).

good understanding of the $\Gamma$ dependence of the flow and the heat transfer for small $\Gamma$ is mandatory. The Göttingen group $[34,39-41,50,53]$ has built large-scale cylindrical cells with $1 \geq \Gamma \geq 1 / 3$ and heights up to $H=2.24 \mathrm{~m}$, filled with pressurized $\mathrm{SF}_{6}$ (with low viscosity and nearly constant $\mathrm{Pr}$ ) and has experimentally studied the onset $\mathrm{Ra}_{u, \Gamma}$ of the ultimate regime in almost-OB RBC. Note that building even larger setups is not prohibitive, but simply extremely costly. The Göttingen group found that the onset occurs at Ra around $10^{14}$ (consistent with the theoretical estimate of Grossmann and Lohse [15]) and revealed a $\Gamma$ dependence as $\operatorname{Ra}_{u, \Gamma} \propto \Gamma^{-3.04}$ [54]; i.e., smaller $\Gamma$ require considerably larger Ra to observe the onset. Also Roche et al. [55,56], for $1.14 \geq \Gamma \geq 0.23$, found a strong $\Gamma$ dependence of $\operatorname{Ra}_{u, \Gamma}$ with the same trend. Based on an analysis of different experimental data [39-43,50,55,57-59], they also proposed that for small $\Gamma$ the onset $\mathrm{Ra}$ for the ultimate regime goes approximately as $\operatorname{Ra}_{u, \Gamma} \sim \Gamma^{-3}$.

In fact, due to the stabilizing effect of the sidewalls in small- $\Gamma$ cells, it is not surprising at all that flow transitions are shifted toward much larger Ra. This already holds at the onset of convection: While without lateral confinement (i.e., $\Gamma \rightarrow \infty$ ) this onset occurs at a critical $\operatorname{Ra}_{c, \infty} \approx 1708$ [60], for small $\Gamma$ the critical $\mathrm{Ra}_{c, \Gamma}$ is much larger [61-71]. In the limit $\Gamma \rightarrow 0$, Catton and Edwards [63] numerically solved the linearized perturbation equations with approximate wall conditions and proposed the scaling $\operatorname{Ra}_{c, \Gamma} \sim \Gamma^{-4}$ for the onset $\mathrm{Ra}_{c, \Gamma}$ in this limit.

In this Letter, we will derive the scaling relation $\operatorname{Ra}_{c, \Gamma} \sim$ $\Gamma^{-4}$ for $\Gamma \rightarrow 0$ and, in fact, generalize it to any $\Gamma$, be it large or small. We will then show that our numerically performed linear stability analysis (LSA) is consistent with the suggested generalized functional dependence of $\mathrm{Ra}_{c, \Gamma}$ on $\Gamma$. Our result can be cast in the form that the relevant length scale in $\mathrm{RBC}$ is

$$
\ell \sim D / \sqrt{\Gamma^{2}+C}=H / \sqrt{1+C / \Gamma^{2}},
$$

with a constant $C$ that depends on the shape of the cell. We then apply this insight to the fully turbulent case and are able to collapse various heat transfer data $\mathrm{Nu}(\mathrm{Ra}, \Gamma)$ from OB experiments and direct numerical simulations (DNSs) for various $1 / 32 \leq \Gamma \leq 32$ onto one universal curve. 
Theoretical background.-We first recall that the mean kinetic energy dissipation rate $\epsilon_{u}$ and the thermal dissipation rate $\epsilon_{\theta}$ fulfil the exact relations $[72,73]$

$$
\begin{gathered}
\epsilon_{u} \equiv \nu\left\langle(\nabla \mathbf{u})^{2}\right\rangle=\alpha g\left\langle u_{z} T\right\rangle=\frac{\nu^{3}}{H^{4}}(\mathrm{Nu}-1) \frac{\mathrm{Ra}}{\operatorname{Pr}^{2}}, \\
\epsilon_{\theta} \equiv \kappa\left\langle(\nabla T)^{2}\right\rangle=\left(\kappa \Delta^{2} / H^{2}\right) \mathrm{Nu} .
\end{gathered}
$$

Decomposing the temperature field as

$$
T \equiv T_{l}+\theta, \quad T_{l}(z) \equiv T_{b}-(z / H) \Delta
$$

and taking into account $\left\langle u_{z}\right\rangle_{z}=0$ for any $z$, one obtains $\left\langle u_{z} T\right\rangle_{z}=\left\langle u_{z} \theta\right\rangle_{z}$ and, hence,

$$
\left\langle u_{z} T\right\rangle=\left\langle u_{z} \theta\right\rangle .
$$

From (4) and (7)-(9), we get

$$
\left\langle u_{z} \theta\right\rangle=(\kappa H / \Delta)\left\langle(\nabla \theta)^{2}\right\rangle
$$

and then with (6) and (1) we obtain

$$
\left\langle(\nabla \mathbf{u})^{2}\right\rangle=\operatorname{Ra}\left[\kappa /\left(\Delta H^{3}\right)\right]\left\langle u_{z} T\right\rangle .
$$

From this, applying successively (9), the Cauchy-Schwarz inequality, and relation (10), we derive

$$
\begin{aligned}
\mathrm{Ra} & =\frac{\Delta H^{3}}{\kappa} \frac{\left\langle(\nabla \mathbf{u})^{2}\right\rangle}{\left\langle u_{z} T\right\rangle}=\frac{\Delta H^{3}}{\kappa} \frac{\left\langle(\nabla \mathbf{u})^{2}\right\rangle\left\langle u_{z} \theta\right\rangle}{\left\langle u_{z} \theta\right\rangle^{2}} \\
& \geq \frac{\Delta H^{3}}{\kappa} \frac{\left\langle(\nabla \mathbf{u})^{2}\right\rangle\left\langle u_{z} \theta\right\rangle}{\left\langle u_{z}^{2}\right\rangle\left\langle\theta^{2}\right\rangle} \geq H^{4} \frac{\left\langle(\nabla \mathbf{u})^{2}\right\rangle\left\langle(\nabla \theta)^{2}\right\rangle}{\left\langle\mathbf{u}^{2}\right\rangle\left\langle\theta^{2}\right\rangle} .
\end{aligned}
$$

For a slightly supercritical $\mathrm{Ra} \gtrsim \mathrm{Ra}_{c, \Gamma}$ the flow is symmetric so that $\langle\mathbf{u}\rangle=0$ and $\langle\theta\rangle=0$ holds. Therefore, we can apply the Poincaré-Friedrichs inequality to the righthand side of (11) to obtain

$$
\operatorname{Ra}_{c, \Gamma} \gtrsim H^{4} \frac{\left\langle(\nabla \mathbf{u})^{2}\right\rangle\left\langle(\nabla \theta)^{2}\right\rangle}{\left\langle\mathbf{u}^{2}\right\rangle\left\langle\theta^{2}\right\rangle} \gtrsim \Lambda^{2},
$$

where $\Lambda$ is the smallest relevant eigenvalue of the Laplacian in a cylindrical domain with a unit height and aspect ratio $\Gamma$, for certain integers $m, n$, and $k$,

$$
\Lambda=m^{2} \pi^{2}+4 \alpha_{n k}^{2} \Gamma^{-2} \sim 1+C \Gamma^{-2} .
$$

For Dirichlet or Neumann boundary conditions, $\alpha_{n k}$ are the first relevant roots of the Bessel function $J_{n}$ or of its derivative, respectively. Under the assumption that the relevant eigenvalues admit positive as well as negative values of $\theta$ and $\mathbf{u}$ in both horizontal and vertical directions, we obtain an estimate of the smallest relevant value of $\Lambda$ for $m=2, n=k=1$, leading to $C \approx 1.49$.
For an infinite fluid layer (or for a cell with an infinite diameter $D$, i.e., $\Gamma \rightarrow \infty) \mathrm{Ra}_{c, \infty} \approx 1708$. Using this, relations (13) and (12), under assumption that $\Gamma$ and $\mathrm{Ra}_{c, \infty}$ are independent parameters, we obtain

$$
\mathrm{Ra}_{c, \Gamma} \sim \operatorname{Ra}_{c, \infty}\left(1+C \Gamma^{-2}\right)^{2}
$$

as estimate for the critical $\mathrm{Ra}_{c, \Gamma}$ for the onset of convection in a container with finite aspect ratio $\Gamma$.

Similarly, we estimate the growth of $\mathrm{Nu}$ near $\mathrm{Ra}_{c, \Gamma}$ from (11), the Poincaré-Friedrichs inequality, and $\left\langle\theta^{2}\right\rangle \leq \Delta^{2}$,

$$
\mathrm{Ra} \geq \Lambda H^{2}\left\langle(\nabla \theta)^{2}\right\rangle /\left\langle\theta^{2}\right\rangle \geq \Lambda H^{2} \Delta^{-2}\left\langle(\nabla \theta)^{2}\right\rangle .
$$

From (8), (7), and (15) we finally obtain $\mathrm{Ra} \geq \Lambda(\mathrm{Nu}-1)$, which, when combined with (13), implies that close to the onset of convection, the Nusselt number behaves as

$$
\mathrm{Nu}-1 \sim\left(1+C \Gamma^{-2}\right)^{-1} \mathrm{Ra} .
$$

From this and the fact that, in the classical turbulent regime (for not too small $\mathrm{Pr}$ and not extremely high $\mathrm{Ra}$ ), $\mathrm{Nu}$ roughly grows as $\sim \mathrm{Ra}^{1 / 3}$, one can expect a collapse of the OB numerical and experimental data for various $\Gamma$, if these are plotted as $f \equiv(\mathrm{Nu}-1) \mathrm{Ra}^{-1 / 3}$ against

$$
\mathrm{Ra}_{\ell} \equiv \mathrm{Ra}\left(1+C \Gamma^{-2}\right)^{-3 / 2}
$$

(for fixed Pr). Close to the onset of convection, this dependence reduces to $f \sim \mathrm{Ra}_{\ell}^{2 / 3}$, while in the developed, statistically steady convective flow $f \sim \mathrm{Ra}_{\ell}^{0} \sim$ const. The variable $\mathrm{Ra}_{\ell}$ is nothing else but a Rayleigh number not based on the cell height $H$, but on the proper length scale $\ell$, Eq. (5). In the limit $\Gamma \rightarrow \infty$, the length scale $\ell$ equals $H$, while for $\Gamma \rightarrow 0$, it is $D$.

Numerical LSA.-We have verified the estimate (14) for the $\Gamma$ dependence of the critical $\mathrm{Ra}_{c, \Gamma}$ for the onset of convection with linearized DNSs for the 2D and 3D cases and with the eigenspectrum LSA for the 2D case. The growth rates obtained with both methods are in a very good agreement, see Figs. 1(a) and 1(b). The numerically obtained $\mathrm{Ra}_{c, \Gamma}$ as function of $\Gamma$ [Eq. (14)] for the isothermal sidewalls are in excellent agreement with the analytical estimates. Equation (14) captures the trend and reflects well also the shape of the neutral curve for the case of adiabatic sidewalls. The best-fit constants $C(C \approx 0.52$ for the $2 \mathrm{D}$ domain and $C \approx 0.77$ for the cylinder) are, however, smaller than the theoretical predictions for the isothermal sidewalls, see Figs. 1(b) and 1(c). Isosurfaces of the temperature of the flow fields near the onset of convection are shown for some $\Gamma$ in Fig. 1 as well. The azimuthalmode transition found for the cylinder between $\Gamma=1$ and 2 is consistent with the experiments [68].

Comparison with heat transfer data from $O B$ experiments and DNS.-Our above theoretical analysis has 
suggested the rescaling $\mathrm{Ra} \rightarrow \mathrm{Ra}_{\ell}$ as a central step to collapse the heat transfer data $\mathrm{Nu}(\mathrm{Ra}, \Gamma)$ for given $\Gamma$, see Eq. (17). This rescaling reflects that the relevant length scale in $\mathrm{RBC}$ for general $\Gamma$ is $\ell \sim D / \sqrt{\Gamma^{2}+C}=$ $H / \sqrt{1+C / \Gamma^{2}}$, see Eq. (5), and not simply the height $H$. For large $\Gamma$ one recovers $\ell=H$, but for small $\Gamma$ one has $\ell=D$. We will now check whether this collapse holds and plot the compensated Nusselt number $f \equiv(\mathrm{Nu}-1) / \mathrm{Ra}^{1 / 3}$ from OB experiments and well-resolved DNSs [74] for various $\Gamma$, both vs $\mathrm{Ra}$ and vs $\mathrm{Ra}_{\ell}$ (with $C=1.49$ ). We do so for two different $\operatorname{Pr}$, namely, for water $(\operatorname{Pr} \approx 4.4$, Fig. 2) and for gas $(\operatorname{Pr} \approx 0.8$, Fig. 3) at room temperature. While in Figs. 2(a) and 3(a) $[f(\mathrm{Ra})]$, the data for small $\Gamma$ show no trend and seem to scatter, in Figs. 2(b) and 3(b) [f( $\left.\left.\mathrm{Ra}_{\ell}\right)\right]$, they nicely collapse on one curve and on the theoretical curve of the unifying theory for turbulent thermal convection [28-30]. A comparison with non-OB data for cryogenic gaseous helium $[42,43,57,75,76]$ is given in the Supplemental Material [9]. As the derivation of the scaling relations is for OB conditions, we do not expect non-OB data to fulfil these relations, and indeed, in general, they do not (see $[34,77,78]$ and Supplemental Material [9]).

Let us now estimate the $\Gamma$ dependence of the onset of the ultimate regime of thermal convection, i.e., $\mathrm{Ra}_{u, \Gamma}$. (The other aspects of the ultimate regime are beyond the scope of this Letter.) The $\Gamma$ dependence of $\operatorname{Ra}_{u, \Gamma}$ has been observed in the Göttingen data [34,39-41,50], with increasing $\mathrm{Ra}_{u, \Gamma}$ for decreasing $1 \geq \Gamma \geq 1 / 3$; see the vertical lines for large Ra in Fig. 3(a). However, as suggested by our theory, in the rescaled Fig. 3(b), these vertical lines collapse at the same $\mathrm{Ra}_{\ell, u} \approx 2.4 \times 10^{13}$. This implies that the $\Gamma$ dependence of $\mathrm{Ra}_{u, \Gamma}$ in the OB case is

$$
\operatorname{Ra}_{u, \Gamma} \approx \operatorname{Ra}_{\ell, u}\left[\Gamma^{2} /\left(C+\Gamma^{2}\right)\right]^{-3 / 2},
$$

which for $\Gamma \ll 1$ simplifies to the estimate $\operatorname{Ra}_{u, \Gamma} \sim \Gamma^{-3}$, in agreement with the experimental data [54]. Note that in Fig. 3 the agreement between the derived relation (18) and measurements is demonstrated for all available almost-OB experimental data, that is, for $\Gamma=1,1 / 2$, and $1 / 3$. Figure 3 and Eq. (18) also show that the presented DNS for small $\Gamma$ by far do not have large enough $\mathrm{Ra}$ to see the expected onset of the ultimate regime.

In conclusion, we have developed a theory to account for the $\Gamma$ dependence of the heat transfer in buoyancy driven convection under OB conditions in cylindrical cells. In particular, we find the $\Gamma$ dependence of the onset of convection $\mathrm{Ra}_{c, \Gamma}$ [Eq. (14), consistent with the LSA] and of the onset of the ultimate regime $\mathrm{Ra}_{u, \Gamma}$ [Eq. (18), consistent with the Göttingen experiments]. Both equations reflect that the relevant length scale in $\mathrm{OB} \mathrm{RBC}$ is $\ell=D / \sqrt{\Gamma^{2}+C}=H / \sqrt{1+C / \Gamma^{2}}$, which only in the limiting cases $\Gamma \rightarrow \infty$ or $\Gamma \rightarrow 0$ become the cell height
$H$ or the cell diameter $D$, respectively. Speaking more generally, our results show how strongly finite-size effects affect scaling relations and that small- $\Gamma$ OB DNSs or (almost) $\mathrm{OB}$ experiments require much large $\mathrm{Ra}$ to achieve the ultimate regime.

The authors acknowledge the Deutsche Forschungsgemeinschaft (SPP1881 "Turbulent Superstructures" and Grants No. Sh405/7, No. Sh405/8, and No. Sh405/ 10), the Twente Max-Planck Center, the European Research Council (ERC Starting Grant No. 804283 UltimateRB), the National Natural Science Foundation of China (Grant No. 91952101), PRACE (Projects No. 2020235589 and No. 2020225335), and the Gauss Centre for Supercomputing e.V. for providing computing time in the GCS Supercomputer SuperMUC at Leibniz Supercomputing Centre.

*Olga.Shishkina@ds.mpg.de

[1] G. Ahlers, S. Grossmann, and D. Lohse, Rev. Mod. Phys. 81, 503 (2009).

[2] D. Lohse and K.-Q. Xia, Annu. Rev. Fluid Mech. 42, 335 (2010).

[3] F. Chillà and J. Schumacher, Eur. Phys. J. E 35, 58 (2012).

[4] E. P. van der Poel, R. Ostilla Mónico, J. Donners, and R. Verzicco, Computers \& Fluids 116, 10 (2015).

[5] V. Theofilis, Annu. Rev. Fluid Mech. 43, 319 (2011).

[6] P. J. Schmid, Annu. Rev. Fluid Mech. 39, 129 (2007).

[7] P. Reiter, X. Zhang, R. Stepanov, and O. Shishkina, J. Fluid Mech. 913, A13 (2021).

[8] P. Reiter, https://github.com/preiter93/pylsa (2021).

[9] See Supplemental Material at http://link.aps.org/ supplemental/10.1103/PhysRevLett.128.084501 for linear stability analysis and comparison with non-OB data, which includes Refs. [10-12].

[10] G. Grötzbach, J. Comput. Phys. 49, 241 (1983).

[11] L. Zwirner, A. Tilgner, and O. Shishkina, Phys. Rev. Lett. 125, 054502 (2020).

[12] K. P. Iyer, J. D. Scheel, J. Schumacher, and K. R. Sreenivasanan, Proc. Natl. Acad. Sci. U.S.A. 117, 7594 (2020).

[13] R. Kraichnan, Phys. Fluids 5, 1374 (1962).

[14] E. A. Spiegel, Annu. Rev. Astron. Astrophys. 9, 323 (1971).

[15] S. Grossmann and D. Lohse, Phys. Fluids 23, 045108 (2011).

[16] R. Hartmann, K. Chong, R. Stevens, R. Verzicco, and D. Lohse, Europhys. Lett. 135, 24004 (2021).

[17] A. Nikolaenko, E. Brown, D. Funfschilling, and G. Ahlers, J. Fluid Mech. 523, 251 (2005).

[18] X. Zhang, R. E. Ecke, and O. Shishkina, J. Fluid Mech. 915, A62 (2021).

[19] S. Weiss and G. Ahlers, J. Fluid Mech. 676, 5 (2011).

[20] R. J. A. M. Stevens, J. Overkamp, D. Lohse, and H. J. H. Clercx, Phys. Rev. E 84, 056313 (2011).

[21] R. J. A. M. Stevens, H. J. H. Clercx, and D. Lohse, Phys. Rev. E 86, 056311 (2012).

[22] D. Funfschilling, E. Brown, A. Nikolaenko, and G. Ahlers, J. Fluid Mech. 536, 145 (2005). 
[23] S. Horn and O. Shishkina, Phys. Fluids 26, 055111 (2014).

[24] J.-Q. Zhong and G. Ahlers, J. Fluid Mech. 665, 300 (2010).

[25] R. Lakkaraju, R. J. A. M. Stevens, R. Verzicco, S. Grossmann, A. Prosperetti, C. Sun, and D. Lohse, Phys. Rev. E 86, 056315 (2012).

[26] O. Shishkina and A. Thess, J. Fluid Mech. 633, 449 (2009).

[27] O. Shishkina, M. S. Emran, S. Grossmann, and D. Lohse, Phys. Rev. Fluids 2, 103502 (2017).

[28] S. Grossmann and D. Lohse, J. Fluid Mech. 407, 27 (2000).

[29] S. Grossmann and D. Lohse, Phys. Rev. Lett. 86, 3316 (2001).

[30] R. J. A. M. Stevens, E. P. van der Poel, S. Grossmann, and D. Lohse, J. Fluid Mech. 730, 295 (2013).

[31] L. Zwirner and O. Shishkina, J. Fluid Mech. 850, 984 (2018).

[32] R. J. A. M. Stevens, D. Lohse, and R. Verzicco, in Direct and Large Eddy Simulation XII, edited by M. GarcíaVillalba, H. Kuerten, and M. Salvetti (Springer, Cham, 2020), Vol. 27, p. 215, 10.1007/978-3-030-42822-8_28.

[33] R. J. A. M. Stevens, A. Blass, X. Zhu, R. Verzicco, and D. Lohse, Phys. Rev. Fluids 3, 041501(R) (2018).

[34] X. He, E. Bodenschatz, and G. Ahlers, J. Fluid Mech. 931, A7 (2022).

[35] J. Bailon-Cuba, M. S. Emran, and J. Schumacher, J. Fluid Mech. 655, 152 (2010).

[36] R. J. A. M. Stevens, R. Verzicco, and D. Lohse, J. Fluid Mech. 643, 495 (2010).

[37] X. Zhang, D. P. M. van Gils, S. Horn, M. Wedi, L. Zwirner, G. Ahlers, R. E. Ecke, S. Weiss, E. Bodenschatz, and O. Shishkina, Phys. Rev. Lett. 124, 084505 (2020).

[38] M. Wedi, D. P. van Gils, S. Weiss, and E. Bodenschatz, J. Fluid Mech. 912, A30 (2021).

[39] G. Ahlers, X. He, D. Funfschilling, and E. Bodenschatz, New J. Phys. 14, 103012 (2012).

[40] X. He, D. Funfschilling, H. Nobach, E. Bodenschatz, and G. Ahlers, Phys. Rev. Lett. 108, 024502 (2012).

[41] X. He, D. Funfschilling, H. Nobach, E. Bodenschatz, and G. Ahlers, Phys. Rev. Lett. 110, 199401 (2013).

[42] X. Chavanne, F. Chilla, B. Castaing, B. Hebral, B. Chabaud, and J. Chaussy, Phys. Rev. Lett. 79, 3648 (1997).

[43] X. Chavanne, F. Chillà, B. Chabaud, B. Castaing, and B. Hébral, Phys. Fluids 13, 1300 (2001).

[44] M. S. Emran and O. Shishkina, J. Fluid Mech. 882, A3 (2020).

[45] J. D. Scheel and J. Schumacher, Phys. Rev. Fluids 2, 123501 (2017).

[46] Data acquired using the small convection apparatus as described in [47].

[47] S. Weiss, P. Wei, and G. Ahlers, Phys. Rev. E 93, 043102 (2016).

[48] G. L. Kooij, M. A. Botchev, E. M. A. Frederix, B. J. Geurts, S. Horn, D. Lohse, E. P. van der Poel, O. Shishkina, R. J. A. M. Stevens, and R. Verzicco, Comput. Fluids 166, 1 (2018).
[49] S. Wagner, O. Shishkina, and C. Wagner, J. Fluid Mech. 697, 336 (2012).

[50] X. He, D. Funfschilling, E. Bodenschatz, and G. Ahlers, New J. Phys. 14, 063030 (2012).

[51] O. Shishkina and C. Wagner, Phys. Fluids 19, 085107 (2007).

[52] O. Shishkina and C. Wagner, J. Fluid Mech. 546, 51 (2006).

[53] X. He, E. Bodenschatz, and G. Ahlers, Proc. Natl. Acad. Sci. U.S.A. 117, 30022 (2020).

[54] E. Bodenschatz, X. He, D. van Gils, and G. Ahlers, 15th European Turbulence Conference (Delft University of Technology, Netherlands, 2015), http://etc15.fyper.com/ proceedings/proceedings/documents/210.pdf.

[55] P.-E. Roche, F. Gauthier, R. Kaiser, and J. Salort, New J. Phys. 12, 085014 (2010).

[56] P.-E. Roche, New J. Phys. 22, 073056 (2020).

[57] J. J. Niemela, L. Skrbek, K. R. Sreenivasan, and R. J. Donnely, Nature (London) 404, 837 (2000).

[58] J. J. Niemela and K. R. Sreenivasan, J. Fluid Mech. 481, 355 (2003).

[59] J. J. Niemela and K. R. Sreenivasan, J. Low Temp. Phys. 143, 163 (2006).

[60] S. Chandrasekhar, Hydrodynamic and Hydromagnetic Stability (Clarendon Press, Oxford, 1961).

[61] G. Charlson and R. Sani, Int. J. Heat Mass Transfer 13, 1479 (1970).

[62] I. Catton, J. Heat Transfer 92, 186 (1970).

[63] I. Catton and D. K. Edwards, AlChE J. 16, 594 (1970).

[64] J. C. Buell and I. Catton, J. Heat Transfer 105, 255 (1983).

[65] J. Mizushima, J. Phys. Soc. Jpn. 64, 2420 (1995).

[66] A. Y. Gelfgat, J. Comput. Phys. 156, 300 (1999).

[67] K. Boronska and L. S. Tuckerman, J. Fluid Mech. 559, 279 (2006).

[68] F. Hebert, R. Hufschmid, J. Scheel, and G. Ahlers, Phys. Rev. E 81, 046318 (2010).

[69] B. F. Wang, D. J. Ma, C. Chen, and D. J. Sun, J. Fluid Mech. 711, 27 (2012).

[70] J. Yu, A. Goldfaden, M. Flagstad, and J. D. Scheel, Phys. Fluids 29, 024107 (2017).

[71] O. Shishkina, Phys. Rev. Fluids 6, 090502 (2021).

[72] L. N. Howard, in Applied Mechanics, edited by H. Görtler (Springer, Berlin, 1966), pp. 1109-1115.

[73] E. Siggia, Annu. Rev. Fluid Mech. 26, 137 (1994).

[74] O. Shishkina, R. J. A. M. Stevens, S. Grossmann, and D. Lohse, New J. Phys. 12, 075022 (2010).

[75] P. Urban, P. Hanzelka, V. Musilova, T. Kralik, M. L. Mantia, A. Srnka, and L. Skrbek, New J. Phys. 16, 053042 (2014).

[76] S. Weiss, X. He, G. Ahlers, E. Bodenschatz, and O. Shishkina, J. Fluid Mech. 851, 374 (2018).

[77] D. D. Gray and A. Giorgini, Int. J. Heat Mass Transfer 19, 545 (1976).

[78] D. Funfschilling, E. Bodenschatz, and G. Ahlers, Phys. Rev. Lett. 103, 014503 (2009). 